\title{
Detection of Biologically Relevant Low-Titer Neutralizing Antibodies Against Adeno-Associated Virus Require Sensitive In Vitro Assays
}

\author{
Anita Kruzik, ${ }^{*}+$ Herwig Koppensteiner, ${ }^{\dagger}$ Damir Fetahagic, Bettina Hartlieb, Sebastian Dorn, \\ Stefan Romeder-Finger, Sogue Coulibaly, Alfred Weber, Werner Hoellriegl, Frank M. Horling, \\ Friedrich Scheiflinger, Birgit M. Reipert, and Maurus de la Rosa
}

Baxalta Innovations $\mathrm{GmbH}$, a member of the Takeda group of companies, Vienna, Austria.

†These authors contributed equally to this work.

Patients with preexisting anti-adeno-associated virus serotype 8 (AAV8) neutralizing antibodies (NAbs) are currently excluded from AAV8 gene therapy trials. Therefore, the assessment of biologically relevant AAV8-NAb titers is critical for product development in gene therapy. However, standardized assays have not been routinely used to determine anti-AAV8-NAb titers, contributing to a wide range of reported antiAAV8 prevalence rates. Using a clinical in vitro NAb assay in a separate study, a higher than expected anti-AAV8-NAb prevalence of about $50 \%$ was found in international cohorts. This comparative study has a translational character, confirming the biological relevance of anti-AAV8-antibody titers measured by this assay. The significance of low-titer anti-AAV8 NAbs is shown, along with the relevance of the in vitro assay cutoff (1:5) compared with other assays. Importantly, internally standardized reagents and purified AAV8 constructs containing $90 \%$ full capsids were used to reduce the effect of empty capsids. It was found that even very low anti-AAV8-NAb titers $(<1: 5)$ could efficiently hinder transduction in vivo, demonstrating the importance of sensitive NAb assays for clinical applications. The in vitro NAb assay was found to be more sensitive than an in vivo NAb assay and thus more suitable for patient screening. Additionally, the study showed that anti-AAV8-NAb titers $<1: 5$ were very rare, further supporting the in vitro assay. However, assays using a lower cutoff may still be useful to explain potential variances in transgene expression. These findings support the relevance of the higher than expected prevalence of anti-AAV8 NAbs, highlighting the need for strategies to circumvent preexisting anti-AAV8 NAbs.

Keywords: AAV, gene therapy, neutralizing antibodies, prevalence, biological relevance, NAb assay

\section{INTRODUCTION}

PREEXISTING ANTI-ADENO-ASSOCIATED VIRUs serotype 8 (AAV8) neutralizing antibodies (NAbs) are a major obstacle to successful AAV8-mediated gene therapy. In both clinical ${ }^{1}$ and nonclinical ${ }^{2,3}$ studies, even low titers of anti-AAV8 NAbs have been shown to reduce the efficiency of transgene expression. Accordingly, patients with detectable preexisting NAbs are excluded from current gene therapy trials, making the reliable assessment of NAbs critical to the success of gene therapy. A current controversy regarding the necessity to determine NAbs in nonclinical AAV studies in non- human primates ${ }^{4-10}$ further emphasizes that a sensitive, standardized assay system using a validated cutoff and standardized controls is required. Such a sensitive and well characterized assay could help the understanding of variances in treatment outcomes among patients receiving gene therapy.

The considerable variation in anti-AAV8-NAb prevalence rates reported across different studies (20-80\%) might reflect not only actual population differences but also assay differences that are indicative of a lack of assay standardization. ${ }^{11} \mathrm{~A}$ comparison of NAb prevalence rates between populations revealed little geographic variability ${ }^{12}$

${ }^{*}$ Correspondence: Anita Kruzik, Donau-City-Strasse 7, 1220 Vienna, Austria. E-mail: anita.kruzik@takeda.com

(c) Anita Kruzik, et al., 2019; Published by Mary Ann Liebert, Inc. This Open Access article is distributed under the terms of the Creative Commons License (http://creativecommons.org/licenses/by/4.0), which permits unrestricted use, distribution, and reproduction in any medium, provided the original work is properly cited. 
(Kruzik et al., 2018, Prevalence of anti-adenoassociated virus immune responses in international cohorts of healthy donors, [in review]), suggesting that inter-assay differences account for most of the observed variation between studies. In general, NAb titers in different studies have been measured using either an in vivo transduction inhibition assay in mice ${ }^{13}$ or cellular in vitro assays. ${ }^{14-16}$ Both types of assay are known to have inherent variability. Known sources of variability for in vitro assays include the full-to-empty capsid ratio of the reporter construct and the starting dilution, which have both been shown to influence the reported NAb prevalence rates strongly. ${ }^{12,17,18}$ In addition, the in vivo assay is more difficult to validate because of a high level of inherent variability between animals. ${ }^{19}$ As a consequence, the clinically relevant prevalence of NAbs against AAV8 and the biological relevance of low antiAAV8-NAb titers remain unclear.

In general, a more comprehensive understanding of the relevance, and hence prevalence, of $\mathrm{NAb}$ titers can be achieved by comparing and modifying different published assay systems using standardized reagents and positive controls to evaluate their effects on NAb prevalence and transgene expression. In addition, the sensitivity of each assay system, and hence its cutoff, has to be assessed carefully because even low anti-AAV8 NAbs are considered to be able to lower transgene expression significantly or block it fully. In this light, studies comparing the sensitivity of in vitro NAb assays to that of in vivo mouse assays have unfortunately so far yielded conflicting results. ${ }^{19-21}$ However, different AAV serotypes were used in these studies, and the lack of standardized reporter constructs used for transduction makes comparisons even more difficult. It was hypothesized that the amounts of empty AAV capsids in the reporter-construct preparation, in particular, may influence the sensitivity of a NAb assay because empty capsids can adsorb NAbs and thereby prevent them from binding to full vector capsids. Accordingly, it has been shown that empty capsids functioning as a decoy for NAbs can significantly impact transduction efficacy. $^{22}$ Therefore, high amounts of empty capsids in reporter construct preparations would decrease the sensitivity of the in vitro NAb assay.

This study has a translational character and aims to assess the biological relevance of the authors' clinical in vitro assay in comparison to different published assays and thereby confirm the average prevalence of anti-AAV8 NAbs (38\%) reported in a separate study (Kruzik et al., 2018, Prevalence of anti-adeno-associated virus immune responses in international cohorts of healthy donors, [in review]), which is higher than expected based on previous reports. ${ }^{11}$ The relevance of the assay and its sensitivity was evaluated by assessing different assay cutoffs and the ability of low NAb titers to interfere with transgene expression. Additionally, the in vitro and in vivo AAV8 NAb assays were evaluated by screening an international cohort of healthy donors and comparing the resulting prevalence reported by each assay. Furthermore, these assays were modified to investigate the effect on the sensitivity and the transgene expression readout of the respective assay systems. To gain a comprehensive understanding of the relevance of low anti-AAV8 NAb and $\mathrm{NAb}$ assays, well characterized reagents and controls were used throughout all assays performed using samples from an international donor cohort.

\section{METHODS}

\section{In vitro NAb assay}

The in vitro NAb assay was performed as described (Kruzik et al., 2018, Prevalence of antiadeno-associated virus immune responses in international cohorts of healthy donors, [in review]). In brief, $\mathrm{HuH} 7$ cells were seeded at $2 \times 10^{4}$ cells per well on 96 -well plates and, after $20-24 \mathrm{~h}$, were infected with the helper virus adenovirus 5. Serial dilutions of samples were generated by diluting them in the respective matrix (human or mouse negative plasma). Human or mouse plasma samples were preincubated with AAV8 luciferase reporter constructs (90\% full capsids; multiplicity of infection [MOI] $3 \times 10^{4}$ ) and then added to the cells. The MOI was optimized during assay development. After 20-24 h, luciferase expression was measured using the ONE-Glo ${ }^{\mathrm{TM}}$ Luciferase Assay System (Promega Corporation, Madison, WI). The NAb titer was defined as the highest dilution that reduced the luciferase expression by $\geq 50 \%$. The assay was validated following regulatory guidelines for clinical studies. ${ }^{23}$ During validation, assay variation was determined to be \pm 1 titer step. Alternatively, starting dilutions of 1:2.5 and 1:1.25 were used.

\section{Healthy donors}

Plasma samples from 60 healthy donors from different regions of Europe were used in this study. The samples were purchased from in.vent Diagnostica GmbH (Berlin, Germany) or collected in Austria, as approved by the ethics committee of the Medical University Vienna. The research was approved by the relevant institutional review boards or ethics committees, and all human participants gave written informed consent. Samples from the 
same donors were used for a prevalence study reported separately (Kruzik et al., 2018, Prevalence of anti-adeno-associated virus immune responses in international cohorts of healthy donors, [in review]).

\section{Reagents}

Monoclonal antibodies. mAb-AAV8-IgG-1 was a purified, human monoclonal neutralizing antiAAV8 antibody generated by phage display (BioRad AbD Serotec GmbH, Puchheim, Germany); ADK8 was a purified mouse monoclonal neutralizing anti-AAV8 antibody (PROGEN Biotechnik GmbH, Heidelberg, Germany).

Purified AAV8 reporter constructs. Purified AAV8 reporter constructs were used: AAV8 luciferase for the in vitro NAb assay, and AAV8 factor IX (FIX) for the in vivo assay (University of North Carolina Gene Therapy Center Vector Core, Chapel Hill, NC). Purified full AAV8-FIX capsids were mixed with empty AAV8 capsids collected from the production process to generate AAV8 FIX with 90\% empty capsids (Baxalta Innovations $\mathrm{GmbH}$, a member of the Takeda group of companies, Vienna, Austria, data on file).

\section{In vivo assay}

All animal experiments were approved by the Institutional Care and Use Committee and Austrian regulatory authorities and were conducted in accordance with Austrian laws on animal experimentation. NOD/SCID (NOD.CB17-Prkdc $\mathrm{scid}^{\mathrm{s}} \mathrm{J}$; Charles River, Saint-Germain-Nuelles, France) mice, 8-10 weeks of age, were used for in vivo studies. As shown in Fig. 1, mice were intravenously injected with $50 \mu \mathrm{L}$ of the NAb sample (intravenous immunoglobulin [IVIG], monoclonal antibody controls, or human plasma). Each treatment group comprised five animals. After $3 \mathrm{~h}$, the mice received $150 \mu \mathrm{L}$ AAV8 FIX $\left(2 \times 10^{10} \mathrm{vg} /\right.$ mouse $)$, as described. ${ }^{13}$ Blood for in vitro NAb analysis was collected by retro-orbital puncture directly before gene therapy. Blood for analysis of FIX expression was collected by cardiac puncture 14 days after gene therapy was applied. Each experiment included control groups injected with AAV8 FIX only or with phosphate-buffered saline (PBS). In the assay modification experiments, NAb samples of $200 \mu \mathrm{L}$ were either injected as described ${ }^{24}$ or preincubated with AAV8 FIX for $1 \mathrm{~h}$.

\section{Detection of FIX protein concentration}

FIX protein concentration was measured with an enzyme-linked immunosorbent assay (ELISA) using commercially available polyclonal-paired anti-human FIX antibodies. Anti-human FIX immunoglobulin G (IgG) was bound to the wells of a 96 -well polystyrene microplate ( $\mathrm{pH}$ 9.6). The coated wells were then blocked ( $0.1 \%$ bovine serum albumin, $2 \mathrm{mM}$ benzamidine, $10 \mathrm{mM}$ EDTA in PBS with $0.05 \%$ Tween 20 ) before the dilution series of standards or samples (comprising several geometric dilutions in duplicate) were loaded into the wells. FIX was captured from the sample by the plateimmobilized antibody and detected by the binding of a peroxidase-labeled anti-human FIX IgG. Finally, peroxidase activity was measured. Samples were diluted to obtain concentrations within the range of the standard curve and were measured in serial dilutions. The ELISA was qualified in accordance with regulatory guidelines. ${ }^{23}$

\section{Data-sharing statement}

Data are the proprietary property of Baxalta Innovations $\mathrm{GmbH}$, a member of the Takeda group of companies, and access can be requested by contacting their legal representative.

\section{RESULTS}

\section{The in vitro NAb assay is more sensitive than the in vivo assay}

To evaluate the relevance of anti-AAV8-NAb titers on the efficiency of gene therapy in vivo, the study tested 60 plasma samples from healthy donors with known in vitro NAb titers in the in vivo NOD/SCID mouse assay. AAV8 constructs

\begin{tabular}{|l|lll|}
\hline $\begin{array}{l}\text { 8- to 12-week-old } \\
\text { NOD/SCID mice }\end{array}$ & $\begin{array}{l}\text { i.v. injection of } \\
\text { IVIG/human } \\
\text { plasma }\end{array}$ & gene therapy \\
\hline
\end{tabular}

Figure 1. Schematic overview of the in vivo assay. NOD/SCID mice aged 8-10 weeks were injected with human plasma or intravenous immunoglobulin (IVIG) to establish neutralizing antibodies (NAbs) in the mouse circulation prior to adeno-associated virus serotype 8 (AAV8)-factor IX (FIX) gene therapy. FIX expression was measured after 14 days. 
with $90 \%$ full capsids were used in both assays to enable assessment of NAbs without the empty capsids negatively impacting assay sensitivity. Applying a cutoff of $50 \%$ reduction of FIX expression in the in vivo assay, a markedly reduced antiAAV8-NAb prevalence of about $20 \%$ was found using the in vivo mouse assay compared with $43 \%$ $\mathrm{NAb}$ prevalence detected with the in vitro $\mathrm{NAb}$ assay for this cohort (Fig. 2A). Comparing the in vivo expression levels with in vitro NAb titers (Fig. 2B) revealed that all samples with in vitro $\mathrm{NAb}$ titers of $\geq 1: 80$ were also positive in the in vivo assay. However, all samples with low in vitro $\mathrm{NAb}$ titers of $1: 5$ or $1: 10$ were not detected in the in vivo assay using 50\% reduction of FIX expression as a cutoff. An in vitro titer of 1:20 could be considered as the "detection limit" of the in vivo assay because these samples show about $50 \%$ reduction of FIX expression (Fig. 2B).

The study also applied a $30 \%$ reduction of FIX expression as an alternative cutoff point to see how a different cutoff would influence the reported prevalence. This slightly enhanced the sensitivity of the in vivo assay (prevalence of $32 \%$ ), but at the cost of unacceptably high variation that would likely preclude reliable use of the assay for patient screening (data not shown). This variation was caused by the high variability of expression levels noted among individual animals in one group (Supplementary Fig. S1). In this experimental context, the in vivo assay had a lower sensitivity than the in vitro NAb assay (Fig. 2B), suggesting that the in vitro NAb assay is more suitable for detecting low-titer anti-AAV8 NAbs.

An attempt was made to enhance the sensitivity of the in vivo assay by preincubating the donor plasma samples from healthy donors with the AAV8 vector before injection to allow neutralization prior to the dilution in the mouse circulation (data not shown). However, this strategy did not result in enhanced sensitivity. Finally, the plasma volume was increased by a factor of 4 and $200 \mu \mathrm{L}$ instead of $50 \mu \mathrm{L}$ plasma per mouse was injected (Fig. 2C), as described. ${ }^{24}$ This modification slightly improved the sensitivity of the in vivo assay, but it was still lower than that of the in vitro NAb assay (Fig. 2C). Together, these data show that modification of the in vivo assay does not enhance the assay sensitivity sufficiently to allow detection of low-titer anti-AAV8 NAbs.
A

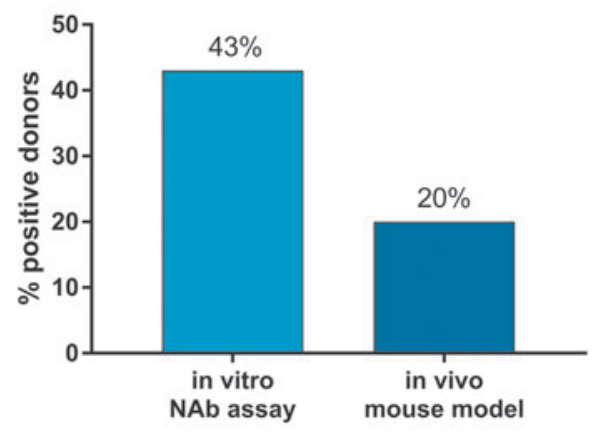

C

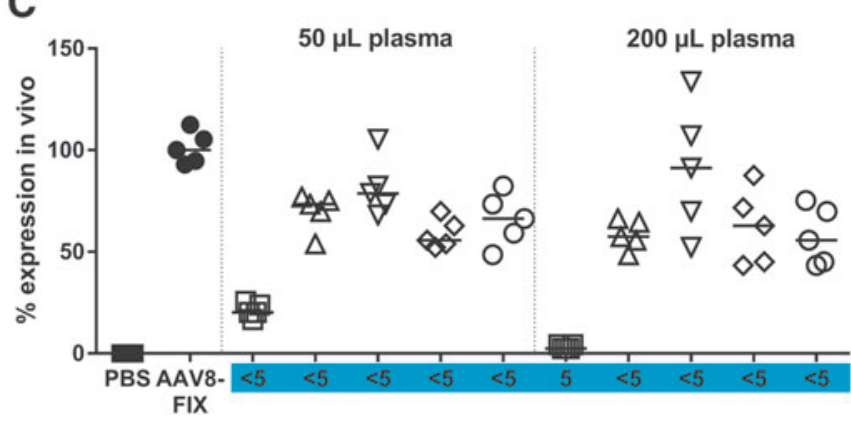

B

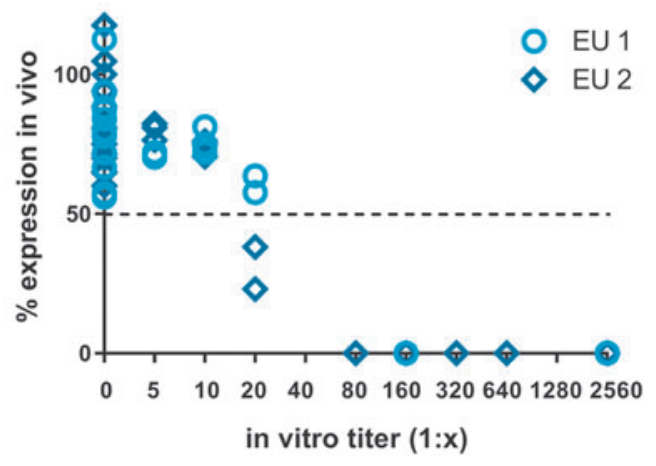

NAb titer in mouse circulation

Figure 2. Comparison of the in vivo assay and the in vitro NAb assay. (A) The NAb prevalence detected using the in vivo assay is lower than that detected using the in vitro NAb assay. (B) Analysis of 60 healthy donors with the in vivo assay. Relative FIX expression compared with the phosphate-buffered saline group is plotted against the in vitro NAb titer of the plasma samples. Each dot represents the median FIX expression of five animals. (C) Mice were injected with $200 \mu \mathrm{L}$ instead of $50 \mu \mathrm{L}$ human plasma. NAb titers in injection samples: $\square, 1: 320 ; \Delta, 1: 10 ; \nabla, 1: 5 ; \diamond, 1: 5 ; \bigcirc,<1: 5$. 


\section{Low-titer NAbs can efficiently block transgene expression in mice}

The biological significance of low anti-AAV8$\mathrm{NAb}$ titers was assessed further, and an explanation for the previous observations (Fig. 2) was sought by determining the sensitivity of the assays more thoroughly using high-titer samples $(>1: 80)$. IVIG (in vitro NAb titer 1:1,280; Fig. 3A), a mixture of antibodies from thousands of plasma donations that contains a mixture of anti-AAV8 NAbs, and high-titer plasma (in vitro NAb titer 1:2,560) from two healthy donors (Fig. 3B) who represented the anti-AAV8-antibody response after natural infection were titrated in the in vivo assay. Prior to gene therapy, mouse plasma samples were collected to analyze the effective anti-AAV8-NAb titers in vivo using the in vitro assay. The dilution of the samples applied in the mouse circulation was calculated to be about 1:70 (based on $25 \mathrm{~g}$ bodyweight, $72 \mathrm{~mL}$ plasma $/ \mathrm{kg}$, and approximately twice the blood volume ${ }^{25}$ ). The NAb titers in the mouse circulation were found to be about eight titer steps lower than in the injected IVIG or human plasma samples (twofold titer steps, dilution factor $=2^{8}=64$; Fig. 3). These data suggest that dilution in the mouse circulation is the reason that low NAb titers in human plasma samples cannot be detected in the in vivo assay.

Transduction was completely blocked in all mice with detectable NAbs $(\geq 1: 5)$ in the circulation. Interestingly, even plasma samples and IVIG with calculated titers $<1: 5$ and up to $1: 4.7$ in mice were also able to block transgene expression completely. Accordingly, no NAbs could be detected in the circulation of these mice prior to gene therapy using the in vitro assay because they were below the detection limit of 1:5 (red boxes in Fig. 3). Moreover, even lower NAb titers were still able to reduce FIX expression significantly by $>50 \%$, showing that low $\mathrm{NAb}$ titers can interfere with transgene expression (blue boxes). The difference between IVIG and donor samples could be explained by the consideration that neutralization by IVIG is potentially more efficient in neutralization of AAV8 than by a single

A

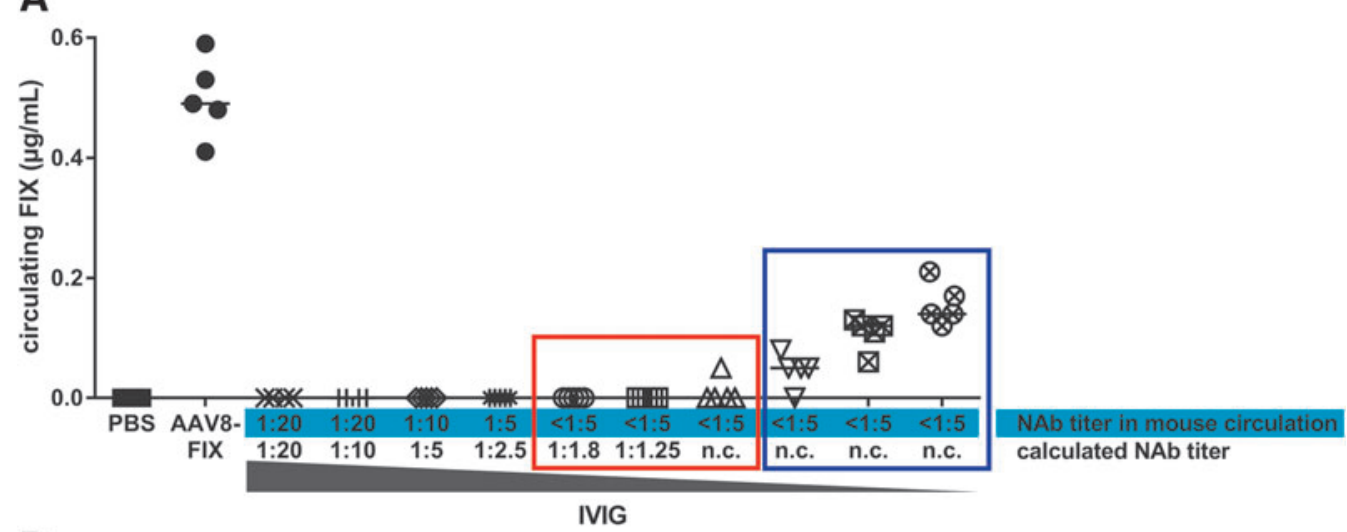

B

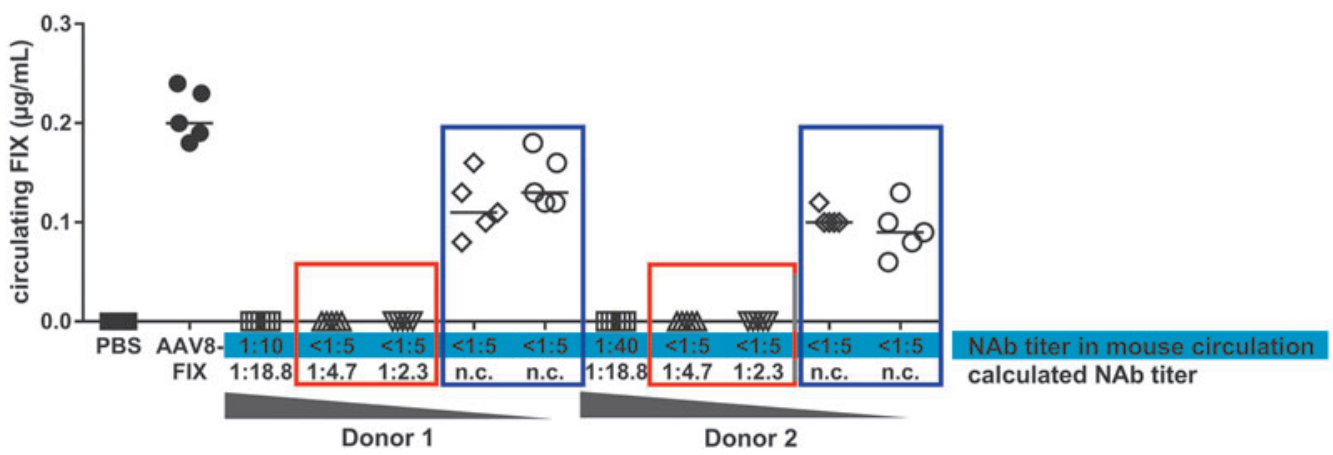

Figure 3. High biological relevance of low NAb titers. FIX expression after mice were injected with different dilutions of (A) IVIG or (B) human plasma with high NAb titers. The $x$-axis shows the NAb titers in the mouse circulation prior to AAV8-FIX injection (blue background, detected NAb titers; white background, calculated NAb titers according to the relative dilution into the mouse blood; NC, not calculated). The boxes indicate NAbs that were not detectable in the mouse circulation but were able to partly (blue boxes) or completely (red boxes) block FIX expression. (A) NAb titers in injected plasma samples: $\square, 1: 1,200$; $\triangle, 1: 300 ; \nabla, 1: 150 ; \diamond, 1: 30 ; \bigcirc, 1: 15$. (B) NAb titers in injected IVIG samples: $\times, 1: 1,280 ;+, 1: 640, \diamond, 1: 320 ;{ }^{*}, 1: 160 ; \bigcirc, 1: 118 ; \square, 1: 80 ; \triangle, 1: 56 ; \nabla, 1: 40 ; \otimes, 1: 20 ;$ $\otimes, 1: 10$. 
high-titer donor plasma, most likely because it contains pooled IgG anti-AAV8 NAbs from multiple donors covering more AAV8 capsid epitopes. These data clearly show that low and borderline NAb titers close to the cutoff in the mouse circulation can be highly efficacious in preventing FIX expression.

\section{Use of monoclonal neutralizing anti-AAV8 antibodies confirms differences in sensitivity between the in vitro NAb assay and the in vivo assay}

IVIG and human plasma are polyclonal antibody reagents each with a different efficacy with regard to AAV8 neutralization, and are not optimal for assay comparison or for assay standardization. For this purpose, purified ADK8, a well characterized and highly neutralizing murine anti-AAV8 antibody, ${ }^{26}$ and mAb-AAV8-IgG-1, a human anti-AAV8 monoclonal antibody (mAb) with lower neutralizing capacity, were used. Both mAbs were diluted to different concentrations, and they were analyzed in the in vivo assay to assess the assay sensitivity (Fig. 4). The results are summarized in Fig. 4C. When assessed with mAb-AAV8-IgG-1, the sensitivity of the in vivo assay was $20 \mu \mathrm{g} / \mathrm{mL}$ (calculated in vitro titer 1:431) compared with $0.232 \mu \mathrm{g} / \mathrm{mL}$ (in vitro titer $1: 5$ ) of the in vitro $\mathrm{NAb}$ assay.
When assessed with mAb ADK8, the sensitivities were $3 \mu \mathrm{g} / \mathrm{mL}$ (calculated in vitro titer 1:392) and $0.038 \mu \mathrm{g} / \mathrm{mL}$ (in vitro titer $1: 5$ ) for the in vivo assay and in vitro $\mathrm{NAb}$ assay, respectively. These data confirm that the in vivo assay is less sensitive than the in vitro NAb assay. For both mAbs, the factor between the sensitivity of the in vitro NAb assay (NAb titer 1:5 at 0.232 and 0.038 , respectively) and the sensitivity of the in vivo assay ( 20 and $3 \mu \mathrm{g} / \mathrm{mL}$, respectively) was around 80 , reflecting roughly the expected dilution in the mouse circulation. The difference in sensitivity assessed by ADK8 and IgG1 can be explained by the different neutralizing capacities of the mAbs and highlights the need for standardized positive controls to compare $\mathrm{NAb}$ assay sensitivities between different laboratories.

\section{Assay modification reveals anti-AAV8-NAb titers $<1: 5$ are rare in human plasma samples}

Figure 3 shows that borderline in vitro NAb titers $(\leq 1: 5)$ in the circulation of mice can block or suppress transgene expression. The starting dilution of the in vitro assay was changed from 1:5 to $1: 2.5$ and $1: 1.25$ to determine the prevalence of potentially significant anti-AAV8-NAb titers $<1: 5$. The high plasma concentration was not toxic to the cells, and the assay was fully functional, as con-
A

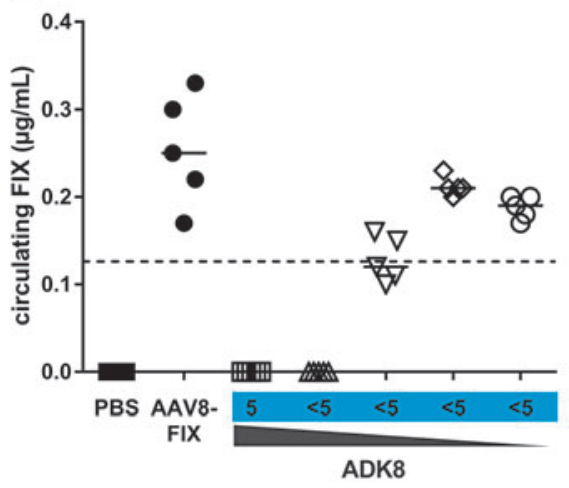

B
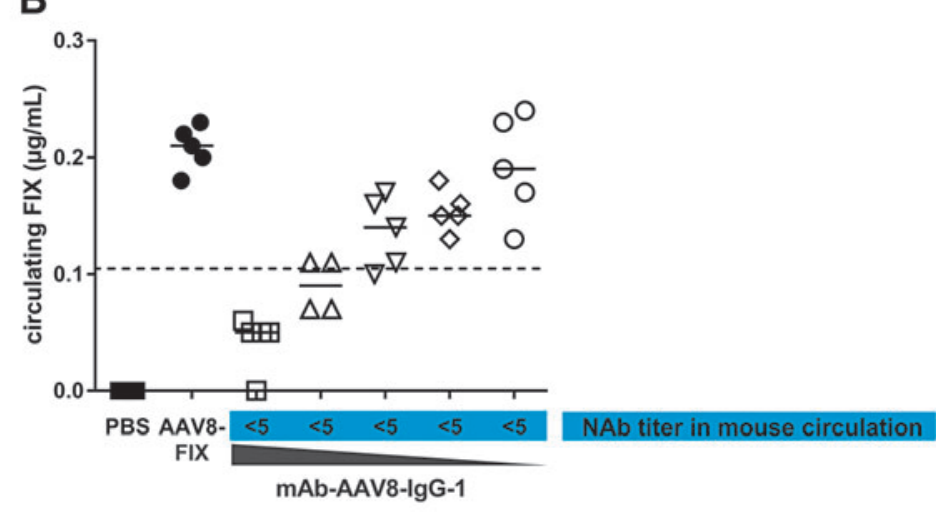

C

The in vivo assay is less sensitive than the in vitro assay

\begin{tabular}{lccc}
\hline Positive control, mAb & NAb titer of stock & $\begin{array}{c}\text { Limit of detection in vitro assay, } \\
\text { NAb titer } 1: 5, \mu \mathrm{g} / \mathrm{mL}\end{array}$ & $\begin{array}{c}\text { Limit of detection } \\
\text { in vivo assay, } \mu \mathrm{g} / \mathrm{mL}\end{array}$ \\
\hline ADK8 & $1: 655360(5 \mathrm{mg} / \mathrm{mL})$ & 0.038 & 2 \\
mAb-AAV8-lgG-1 & $1: 40960(1.9 \mathrm{mg} / \mathrm{mL})$ & 0.232 & 20 \\
\hline
\end{tabular}

The sensitivity of the in vivo assay and the in vitro NAb assay was determined by 2 mAbs with different neutralizing capacities.

Figure 4. Sensitivity of the in vivo assay. The sensitivity of the in vivo assay was determined by analysis of different concentrations of the monoclonal antibodies (mAbs) (A) ADK8 and (B) mAb-AAV8-IgG1. Sensitivity was defined as the lowest mAb concentration that reduced the FIX expression by at least $50 \%$. One representative experiment is shown. Concentration of mAbs in injection samples: (A) $\square, 30 \mu \mathrm{g} / \mathrm{mL}$ (NAb titer 1:3,921); $\triangle, 10 \mu \mathrm{g} / \mathrm{mL}$ (NAb titer 1:1,308); $\nabla, 3 \mu \mathrm{g} / \mathrm{mL}$ (NAb titer 1:392); $\diamond, 1 \mu \mathrm{g} / \mathrm{mL}$ (NAb titer 1:131); $\bigcirc, 0.3 \mu \mathrm{g} / \mathrm{mL}$ (NAb titer 1:39); (B) $\square, 30 \mu \mathrm{g} / \mathrm{mL}$ (NAb titer 1:650); $\triangle, 20 \mu \mathrm{g} / \mathrm{mL}$ (NAb titer 1:431); $\nabla, 10 \mu \mathrm{g} / \mathrm{mL}$ (NAb titer 1:216); $\diamond, 5 \mu \mathrm{g} / \mathrm{mL}$ (NAb titer 1:108); $\bigcirc, 2 \mu \mathrm{g} / \mathrm{mL}$ (NAb titer 1:43). (C) The sensitivity of the in vivo assay and the in vitro NAb assay was determined by 2 mAbs with different neutralizing capacities. 


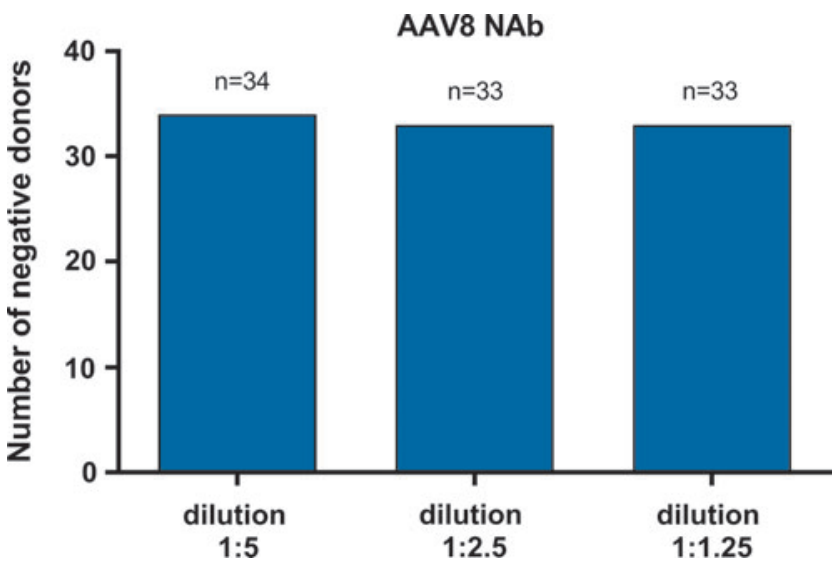

Figure 5. In vitro assay modification. NAb titers $<1: 5$ occur only very rarely. Plasma samples of 34 donors who were negative for NAb in the in vitro NAb assay were reanalyzed with lower starting dilutions. Only one donor was positive at a starting dilution of 1:1.25. The same donor was positive at a starting dilution of 1:2.5.

firmed by results from the positive control, negative control, and positive donors with different NAb titers (data not shown). All 34 donors from the international cohort (Fig. 2B) who were NAb negative were selected, and they were screened in this modified assay. Only one of these donors was positive in the lower starting dilutions (Fig. 5). This donor was clearly negative in the in vivo assay (94\% FIX expression). Lowering the starting dilutions of the in vitro assay to 1:1.25 changed the reported anti-AAV8-NAb prevalence only slightly (from $43 \%$ to $45 \%$ ), suggesting that these lower NAb titers occur very rarely among the international cohort sampled.

\section{Empty capsids bind anti-AAV8 NAbs and thereby influence assay sensitivity}

The impact of empty capsids in the vector preparation on the results of gene therapy in the pres-

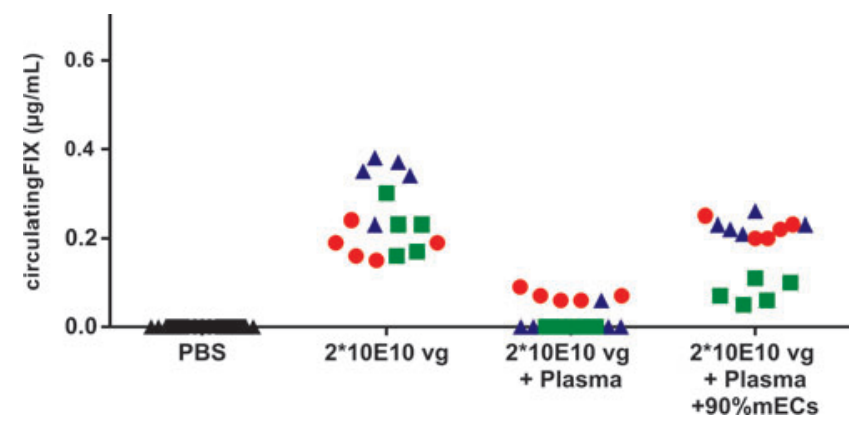

Figure 6. Overcoming low NAb titers with higher doses of empty capsids. Mice were injected with diluted human plasma to establish a low NAb titer of 1:5. The mice received gene therapy with an AAV8-FIX preparation containing only $10 \%$ or $90 \%$ empty capsids. $\boldsymbol{\Lambda}$, group A: plasma sample A; $\boldsymbol{\square}$, group B: plasma sample B; $\bullet$, group $C$, plasma sample C. ence of low NAb titers was assessed using the in vivo $\mathrm{NAb}$ mouse assay. For this purpose, a NAb titer of 1:5 in mice was established using passive transfer of plasma from donors with high NAb titers. The influence of empty capsids was evaluated by the level of FIX transgene expression after vector application in the presence of these low NAb titers and vector preparations containing $90 \%$ empty capsids. At a dose of $2 \times 10^{10} \mathrm{vg} /$ mouse, FIX expression was blocked by NAbs (titer 1:5) in the absence of empty capsids, but FIX expression was partly (donor A, donor B) or fully (donor C) restored by $90 \%$ empty capsids (Fig. 6), showing that the purity of the AAV8FIX preparations determines the level of transgene expression and may affect assay sensitivity.

\section{DISCUSSION}

Multiple studies have been performed so far to determine the preexisting immunity to AAV8. The presumed lower preexisting immunity against AAV8 compared with preexisting immunity to $\mathrm{AAV}^{1}{ }^{1}$ suggests that more patients could potentially benefit from gene therapy treatment, and this was therefore one reason for the development of AAV8 capsids for gene therapy. However, these previous studies suggest a wide prevalence range for anti-AAV8 NAbs: the majority suggest a prevalence rate of 20-30\%, while some studies suggest a higher prevalence rate of $>80 \% .^{11,27-29}$ As there are currently no standardized NAb assays, the comparability and prevalence of reported NAb data are unclear. Furthermore, the biological-and hence potential clinical-relevance of these data remains elusive. The lack of standardized assays and accurate description of NAb assays is highlighted by a current controversy regarding the determination of NAbs in four recent AAV studies in nonhuman primates. ${ }^{4-10}$ The current study can be seen as a first step toward a standardized NAb assay with standardized reagents.

A full evaluation of the assay system requires studies that compare different assay formats and sensitivities. Several factors, including cell line, multiplicity of infection, and reporter constructs and their purity, determine the sensitivity of an in vitro NAb assay. ${ }^{20,30,31}$ Furthermore, the starting dilution (cutoff), which defines the sensitivity of the in vitro assay, has the strongest influence on the NAb titers measured and thus on the NAb prevalence determined with a given assay. As an example highlighting the influence of the assay cutoff on reported prevalence, the use of a 1:20 starting dilution in an in vitro NAb assay resulted in an anti-AAV8-NAb prevalence of $19 \%$ in one 
study, ${ }^{17}$ whereas when the same cutoff (1:20 instead of 1:5) was applied to the present data, the prevalence was reduced from $43 \%$ to $23 \%$. It was demonstrated that low and borderline NAb titers can block or suppress FIX expression efficiently and are therefore highly relevant, indicating that the detection of low anti-AAV8 NAb titers and assay sensitivity are paramount. These observations are in line with the findings of other studies. ${ }^{2,32,33}$

Screening a large international cohort of donors using purified AAV8 FIX to minimize the impact of empty capsids revealed that the in vivo assay is less sensitive than the in vitro assay and is not capable of detecting low anti-AAV8 NAb titers, even after modification. The low sensitivity of the in vivo assay is caused by the dilution of the plasma samples in the mouse circulation. This renders the in vitro assay more suitable for patient screening. Furthermore, critical assays for patient screening should be robust and show little variation. Less variability was observed with the in vitro NAb assay than with the in vivo assay. Similar findings were also reported for an in vivo assay of anti-AAV5 NAbs. ${ }^{19} \mathrm{By}$ contrast, similar in vivo assays were described to be more sensitive than an in vitro NAb assay in previous studies. ${ }^{21,24}$ However, the in vitro assay used in one example ${ }^{24}$ had a 1:20 cutoff. The application of a 1:5 cutoff to the in vitro assay in the same study resulted in similar sensitivity to the in vivo assay. The remaining difference between the reported assay and the assay in this study could be explained by other factors. For instance, the proportion of empty capsids in the vector constructs was not stated but may have influenced the sensitivity. ${ }^{22}$

It was found that even very low NAb titers $(<1: 5)$ derived from high-titer donors were capable of significantly affecting transgene expression in vivo. Importantly, it was shown that anti-AAV8-NAb titers below the cutoff of 1:5 of the in vitro assay are very rare $(2.9 \%)$, confirming that $1: 5$ is a valid assay cutoff for a clinical assay. However, as NAb titers $<1: 5$ may occur in rare cases, it may be useful to apply an exploratory in vitro NAb assay with a lower cutoff in circumstances such as investigating variations in the efficacy of transgene expression.

Because even low NAb titers of 1:5 are biologically highly relevant, studies using a higher cutoff to determine NAbs (cutoff 1:20 ${ }^{12,17}$ ) have the potential to underestimate their prevalence considerably. Moreover, in a recent clinical trial, ${ }^{13}$ the in vivo assay used to screen patients was similar to the in vivo assay used in this study, suggesting that the trial may have included patients with low NAb titers that were not detected by the in vivo assay. Speculating that the full capsids were not purified for the clinical trial, the majority of the AAV8-FIX preparation may have been empty capsids (potentially up to $90 \%$ ). Given that FIX was expressed by all patients in the trial, ${ }^{13}$ it could be hypothesized that the use of $90 \%$ empty capsids in the vector preparation could have resulted in an amelioration of the efficacy of low $\mathrm{NAb}$ titers. Accordingly, this study shows that low NAb titers could be overcome with empty AAV8 capsids in NOD-SCID mice. The data might suggest that empty capsids in reporter construct preparations and in vivo assays can influence the assay sensitivity and could result in a different prevalence, and might be a source of potential variations in transgene expression in clinical trials.

Additionally, these data could be interpreted as suggesting that empty capsids can be used to overcome low anti-AAV8-NAb titers in patients. However, empty capsids in the gene therapy vector may enable treatment of some, but not all, donors with low NAb titers. Furthermore, the efficacy might vary considerably, as suggested by the data using inbred mice. This is in line with nonclinical data from mice ${ }^{33}$ and nonhuman primates. ${ }^{22}$

In summary, this study provides a comprehensive understanding of the performance of different published NAb assays and the authors' clinical assay regarding the detection of low titers of NAbs using well characterized reagents and samples from large international cohorts. The data indicate that lowtiter NAbs are relevant and that highly sensitive $\mathrm{NAb}$ assays are required to detect them. The study shows that the validated clinical NAb assay possesses the sensitivity to detect these biologically relevant low antibody titers. Furthermore, the results presented confirm the higher than expected average prevalence rate of $38 \%$ found in the international cohort of healthy donors (Kruzik et al., 2018, Prevalence of anti-adeno-associated virus immune responses in international cohorts of healthy donors, [in review]). This higher than expected prevalence indicates that a significant proportion of patients cannot be treated, highlighting the need to develop an appropriate dosing strategy for these patients.

\section{ACKNOWLEDGMENTS}

The authors would like to thank Hanspeter Rottensteiner, Maria Schuster, Markus Weiller, Claudia Gold, Karld Schmid, Christian Fiedler, Dominik Mittergradnegger, and Elise Langdon-Neuner. A.K. is $\mathrm{PhD}$ candidate at the Medical University Vienna. This work is submitted in partial fulfilment of the requirement for the $\mathrm{PhD}$. This research was funded by Baxalta Innovations $\mathrm{GmbH}$, a member of the Takeda group of companies, Vienna, Austria. Editing support for this manuscript was provided by 
Isobel Lever, $\mathrm{PhD}$, employee of Excel Medical Affairs (Southport, CT), and was funded by Baxalta. US Inc., a member of the Takeda group of companies, Lexington, MA, USA.

\section{AUTHOR DISCLOSURE}

All authors were employees of Baxalta Innovations $\mathrm{GmbH}$, a member of the Takeda group of companies, Vienna, Austria at the time of the current analysis. A.K., B.H., S.R.F., and A.W. are current employees of Baxalta Innovations $\mathrm{GmbH}$, a member of the Takeda group of companies, Vienna, Austria. W.H., F.S., B.M.R., and M. de la R. are current employees of Baxalta Innovations $\mathrm{GmbH}$, a member of the Takeda group of companies, Vienna, Austria and are stockholders in Takeda Pharmaceutical Company Limited.

\section{SUPPLEMENTARY MATERIAL}

\section{Supplementary Figure S1}

\section{REFERENCES}

1. Manno CS, Pierce GF, Arruda VR, et al. Successful transduction of liver in hemophilia by AAV-Factor $\mathrm{IX}$ and limitations imposed by the host immune response. Nat Med 2006;12:342-347.

2. Wang $L$, Calcedo R, Bell $P$, et al. Impact of preexisting immunity on gene transfer to nonhuman primate liver with adeno-associated virus 8 vectors. Hum Gene Ther 2011;22:1389-1401.

3. Jiang $H$, Couto LB, Patarroyo-White $S$, et al. Effects of transient immunosuppression on adenoassociated, virus-mediated, liver-directed gene transfer in rhesus macaques and implications for human gene therapy. Blood 2006;108:3321-3328.

4. Wang PX, Zhao GN, Ji YX, et al. Wang et al. reply. Nat Med 2018;24:700-701.

5. Zhang P, Wang PX, Zhao LP, et al. The deubiquitinating enzyme TNFAIP3 mediates inactivation of hepatic ASK1 and ameliorates nonalcoholic steatohepatitis. Nat Med 2018;24:84-94.

6. Zhao GN, Zhang P, Gong J, et al. Tmbim1 is a multivesicular body regulator that protects against non-alcoholic fatty liver disease in mice and monkeys by targeting the lysosomal degradation of Tlr4. Nat Med 2017;23:742-752

7. Ji YX, Huang $Z$, Yang $X$, et al. The deubiquitinating enzyme cylindromatosis mitigates nonalcoholic steatohepatitis. Nat Med 2018;24:213-223.

8. Xiao W, Gao G, Ling C, Herzog RW, Xiao X, Samulski RJ. Impact of neutralizing antibodies against $A A V$ is a key consideration in gene transfer to nonhuman primates. Nat Med 2018;24:699.

9. Mingozzi F. AAV immunogenicity: a matter of sensitivity. Mol Ther 2018;26:2335-2336.

10. Wang PX, Ji YX, Zhang XJ, et al. Targeting CASP8 and FADD-like apoptosis regulator ameliorates nonalcoholic steatohepatitis in mice and nonhuman primates. Nat Med 2017;23:439-449.

11. Louis Jeune V, Joergensen JA, Hajjar RJ, et al. Pre-existing anti-adeno-associated virus antibodies as a challenge in AAV gene therapy. Hum Gene Ther Methods 2013;24:59-67.

12. Calcedo R, Vandenberghe $L H, G a o ~ G$, et al. Worldwide epidemiology of neutralizing antibodies to adeno-associated viruses. J Infect Dis 2009;199:381-390.
13. Nathwani AC, Tuddenham EG, Rangarajan S, et al. Adenovirus-associated virus vector-mediated gene transfer in hemophilia B. N Engl J Med 2011;365: 2357-2365.

14. Monahan PE. Gene therapy in an era of emerging treatment options for hemophilia B. J Thromb Haemost 2015;13:S151-160.

15. George LA, Sullivan SK, Giermasz A, et al. Hemophilia $B$ gene therapy with a high-specificactivity factor IX variant. N Engl J Med 2017;377: 2215-2227.

16. Rangarajan S, Walsh L, Lester W, et al. AAV5factor VIII gene transfer in severe hemophilia A. N Engl J Med 2017;377:2519-2530.

17. Boutin S, Monteilhet V, Veron P, et al. Prevalence of serum $\lg G$ and neutralizing factors against adeno-associated virus (AAV) types 1, 2, 5, 6, 8, and 9 in the healthy population: implications for gene therapy using AAV vectors. Hum Gene Ther 2010;21:704-712.

18. Li C, Narkbunnam N, Samulski RJ, et al. Neutralizing antibodies against adeno-associated virus examined prospectively in pediatric patients with hemophilia. Gene Ther 2012;19:288-294.

19. Falese L, Sandza K, Yates B, et al. Strategy to detect pre-existing immunity to AAV gene therapy. Gene Ther 2017;24:768-778.

20. Wang M, Crosby A, Hastie E, et al. Prediction of adeno-associated virus neutralizing antibody activity for clinical application. Gene Ther 2015;22:984-992.

21. Wang D, Zhong L, Li M, et al. Adeno-associated virus neutralizing antibodies in large animals and their impact on brain intraparenchymal gene transfer. Mol Ther Methods Clin Dev 2018;11:65-72.

22. Mingozzi F, Anguela XM, Pavani G, et al. Overcoming preexisting humoral immunity to AAV using capsid decoys. Sci Transl Med 2013;5: $194 r a 192$.

23. European Medicines Agency. Guideline on bioanalytical method validation. EMEA/CHMP/EWP/ 192217/2009). www.ema.europa.eu/docs/en_GB/ document_library/Scientific_guideline/2011/08/WC 500109686.pdf (last accessed March 14, 2019).

24. Wang $L$, Calcedo $R$, Wang $H$, et al. The pleiotropic effects of natural AAV infections on liver-directed gene transfer in macaques. Mol Ther 2010;18: 126-134.

25. Diehl KH, Hull R, Morton D, et al. A good practice guide to the administration of substances and removal of blood, including routes and volumes. J Appl Toxicol 2001;21:15-23.

26. Gurda BL, Raupp C, Popa-Wagner R, et al. Mapping a neutralizing epitope onto the capsid of adeno-associated virus serotype 8. J Virol 2012; 86:7739-7751.

27. Liu 0 , Huang $W$, Zhang $H$, et al. Neutralizing antibodies against AAV2, AAV5 and AAV8 in healthy and HIV-1-infected subjects in China: implications for gene therapy using AAV vectors. Gene Ther 2014;21:732-738.

28. Ling C, Wang $Y$, Feng $Y L$, et al. Prevalence of neutralizing antibodies against liver-tropic adenoassociated virus serotype vectors in 100 healthy Chinese and its potential relation to body constitutions. J Integr Med 2015;13:341-346.

29. van der Marel S, Comijn EM, Verspaget HW, et al. Neutralizing antibodies against adeno-associated viruses in inflammatory bowel disease patients: implications for gene therapy. Inflamm Bowel Dis 2011;17:2436-2442.

30. Meliani A, Leborgne C, Triffault S, et al. Determination of anti-adeno-associated virus vector neutralizing antibody titer with an in vitro reporter system. Hum Gene Ther Methods 2015;26:45-53.

31. Ellsworth JL, O'Callaghan M, Rubin H, et al. Low seroprevalence of neutralizing antibodies targeting two clade F AAV in humans. Hum Gene Ther Clin Dev 2018;29:60-67.

32. Lin J, Calcedo R, Vandenberghe LH, et al. Impact of preexisting vector immunity on the efficacy of adeno-associated virus-based HIV-1 Gag vaccines. Hum Gene Ther 2008;19:663-669.

33. Scallan CD, Jiang $H$, Liu $T$, et al. Human immunoglobulin inhibits liver transduction by AAV vectors at low AAV2 neutralizing titers in SCID mice. Blood 2006;107:1810-1817.

Received for publication December 19, 2018; accepted after revision February 6, 2019.

Published online: February 7, 2019. 